\title{
Comparación del Grado de Satisfacción del Profesorado de Educación Física con la Formación Inicial y la Inserción Profesional
}

\author{
Comparison between the Satisfaction Degree of Physical \\ Education Teachers with Initial Training and Labour \\ Integration
}

\author{
Miriam Sonlleva Velasco * \\ Suyapa Martínez Scott \\ Roberto Monjas Aguado \\ Universidad de Valladolid, España
}

\begin{abstract}
La evaluación de la satisfacción de los docentes con la carrera realizada es uno de los parámetros que permiten medir la calidad de la enseñanza y mejorar el acceso a la función docente. El presente estudio trata de analizar cuál es la satisfacción del profesorado de Educación Física con la carrera realizada atendiendo a tres factores que son decisivos para el diagnóstico: la valoración de la formación inicial recibida, el acceso al mundo laboral y las experiencias profesionales. Desde estos ejes evaluamos —con una metodología mixta - las diferencias que existen entre la satisfacción de hombres y mujeres y aquellas que nacen de las opiniones de egresados de Magisterio de Educación Física y titulados de Ciencias de la Actividad Física y el Deporte. Los resultados muestran cómo la variable "sexo" nos lleva a concluir que no existen diferencias reveladoras entre la satisfacción de hombres y mujeres con la carrera, a pesar de que las motivaciones y percepciones de cada colectivo presentan variaciones. Estas diferencias son mayores atendiendo a la variable "estudios". Las competencias prácticas en la formación recibida, los problemas de acceso a la función docente y las sensaciones negativas percibidas en los primeros destinos son algunos de los aspectos que llevan a los maestros a sentirse menos satisfechos con la carrera. El estudio concluye con la necesidad de profundizar en las barreras que hombres y mujeres tienen en la profesión y conocer si las diferencias en la satisfacción en función de los estudios aumentan con los años de experiencia.
\end{abstract}

Palabras clave. Satisfacción profesional; Educación física; Formación docente; Práctica pedagógica; Evaluación.

\begin{abstract}
The assessment of teachers' satisfaction with their careers is one of the parameters that can be used to measure the quality of teaching and improve access to the teaching function. The present study tries to analyze the satisfaction of Physical Education teachers with the career, taking into account three factors are decisive for the diagnosis: the assessment of the initial training received, access to the world life and professional experiences. From these axes we evaluate with mixed methodology the differences that exist between the satisfaction of men and women and those that come from the opinions of Physical Education Teaching degree graduates and graduates of Sciences of Physical Activity and Sport. The results show how the variable "sex" leads us to conclude that there are no significant differences between the satisfaction of men and women with the career, despite the fact that the motivations and perceptions of each group vary. These differences are significant considering the variable "studies". The practical skills in the training received, the problems of access to the teaching function and the negative sensations perceived in the first destinations are some of the aspects that lead teachers to feel less satisfied with the career. The study concludes with the need to delve deeper into the barriers that men and women have in the profession and to know whether the differences in satisfaction according to studies increase with years of experience.
\end{abstract}

Keywords. Professional satisfaction; Physical education; Teacher training; Pedagogic practice; Assessment.

*Contacto: miriam.sonlleva@uva.es

issn: $1989-0397$

www.rinace.net/riee/

https://revistas.uam.es/riee
Recibido: $\quad 15$ de enero de 2019

$1^{\text {a }}$ Evaluación: 04 de febrero de 2019

$2^{\text {a }}$ Evaluación: 21 de febrero de 2019

Aceptado: $\quad 25$ de febrero de 2019 


\section{Introducción}

En el panorama actual español, con una gran masificación de estudiantes en el sistema universitario y una alta tasa de desempleo entre las personas tituladas, se presenta una gran inquietud por mejorar la calidad docente y el acceso al mundo laboral.

Dentro del ámbito de la educación, desde hace algunos años, son muchos los estudios que se están haciendo eco sobre esta realidad y que consideran la necesidad de conocer las opiniones, pensamientos e intereses de los docentes como un elemento crucial para poder realizar una evaluación de la enseñanza (Edmons, 1982; Jiménez, Terríquez y Robles, 2001; Sander, Stevenson, King y Coates, 2000; Zurita, Viciana, Garofano, Padial y Cepero, 2017). De estos estudios se desprende que la retroalimentación por parte de los estudiantes y los egresados, tanto durante el proceso de formación como una vez terminada la carrera, supone una forma de acercamiento a la realidad formativa y profesional desde la óptica de sus principales beneficiarios y aporta elementos de reflexión útiles para optimizar la formación ofrecida (Gil-Flores et al., 2009).

Esta línea de trabajo ha sido tenida en cuenta en investigaciones recientes dentro del área de Educación Física. Así, hemos podido comprobar que existen abundantes estudios en los últimos años que tratan de recuperar la percepción de los estudiantes en su formación inicial y la de los egresados, sobre diferentes aspectos del proceso educativo (Herranz y López, 2017; Hortigüela y Pérez, 2016; Hortigüela, Abella y Pérez, 2015; López y Palacios, 2012; Martínez, Santos y Castejón, 2017; Sonlleva, Martínez y Monjas, 2018, entre otros).

Estos estudios desvelan que la especialidad de Educación Física, tanto a nivel académico como social, muestra ciertos aspectos comunes con otras especialidades de la titulación del Magisterio, como por ejemplo, el compartir ciertas asignaturas y soportar la presión descalificadora de la sociedad post moderna; pero también permiten ver cómo el profesor de Educación Física tiene ciertas características que le diferencian del resto del profesorado (Moreno, Sicilia y Águila, 2000). Se trata de un profesional que juega un papel crucial en el desarrollo psicológico, afectivo y psicomotriz del estudiante. Además, la relación que establece este profesional con su alumnado resulta ser especial, pues a través de su trabajo es capaz de conocer mejor a sus estudiantes, fomentar sus motivaciones y aportarles un mayor nivel de bienestar (Iznaola y Gabriel, 2008).

Pero investigaciones como la de Gambau (2015), revelan como el estatus marginal de la Educación Física, las deficiencias en la formación del profesorado, la falta de incentivos a los docentes, la dificultad para el desarrollo profesional continuo y la ausencia de recursos son algunas problemáticas que hacen perder a los docentes de esta área la motivación por la carrera. Esta perdida de motivación, repercute directamente en la experiencia de aprendizaje que se ofrece al alumnado a través de las clases de esta materia.

Los aspectos mencionados no solo redundan en la enseñanza, sino que también inciden en la satisfacción del docente con la carrera realizada. Explican Bimbela et al. (2010), que la satisfacción profesional es uno de los indicadores más importantes para llegar a descubrir cuál es la actitud general de los docentes en el aula. La evaluación de este indicador es fundamental para poder identificar las variables que puedan ser modificadas e incentivar la motivación, el bienestar y la satisfacción del profesional por el trabajo (Griffin, 2010). 
Estas conclusiones nos llevan a preguntarnos qué factores condicionan la satisfacción laboral dentro de la profesión docente y a conocer los estudios que se han realizado sobre esta problemática dentro del campo de la Educación Física.

\subsection{La satisfacción profesional, un aspecto clave para valorar la carrera de Educación Física}

La satisfacción profesional viene siendo en los últimos años uno de los temas de mayor interés a nivel científico (Caballero, 2002). Este aspecto es definido como el conjunto de reacciones y sentimientos que el trabajador tiene en la institución en la que desempeña su labor y que le lleva a tener una actitud positiva o negativa con el empleo (Robbins, 1987; Weinert, 1987).

Para Padrón (1994), la satisfacción profesional tiene una estrecha vinculación con la salud mental y el equilibrio personal y no solo viene condicionada por la vivencia subjetiva experimentada por el trabajador, sino que en ella inciden múltiples factores.

En el caso de la profesión docente, la mayoría de los estudios clasifican estos factores en internos y externos. Palomares (2000) detalla que entre los primeros se encuentran el reconocimiento profesional y la motivación vocacional; y en relación a los factores externos se presentarían las condiciones económicas y el estatus social.

Intentando profundizar en estos factores y haciendo una clasificación más detallada, autores como González y Armesto (2012) apuntan que la satisfacción del profesorado con la carrera realizada se puede describir a través de seis ámbitos: a) satisfacción general (prestigio, reconocimiento social, promoción profesional y económica, autonomía personal); b) condiciones de vida en el centro (relaciones con directivos, familias y alumnado; coordinación con otros docentes; participación en la gestión del centro); c) cuestiones académico curriculares (adecuación del currículo a la práctica, organización horaria, formación didáctica, empleo de materiales y recursos); d) aspectos profesionales (acceso a la función docente, especialización, estatus administrativo y posibilidades de reciclaje); e) planificación y puesta en práctica de las actividades del aula (programación, actividades, clima del aula y evaluación); y f) percepción del rendimiento del alumnado).

Además de estos factores, estudios como el de Cabrera, Weerts y Zulick (2004) detallan cómo la satisfacción con la formación recibida en la universidad es uno de los aspectos que han sido más investigados en las encuestas de egresados de otros países, para determinar el grado de satisfacción profesional. Este factor es de gran importancia, pues resulta ser clave, además, para valorar la calidad de la educación ofrecida y conocer los modelos y estrategias que se están ofreciendo al profesional en formación (Zurita et al., 2017).

Algunos de estos índices de satisfacción han sido medidos tanto a través de cuestionarios estandarizados (Carbonero, Crespo, Martín, Ortega y Sánchez, 1996; Griffin, 2010; Miles, 2010; Thompson, 2008), como a través de diferentes tipos de encuestas (Díaz y Barroso; 2008; Fernández, 1993; González y Armesto 2012; Iznaola y Gabriel, 2008; Moreno, Sicilia y Águila, 2000; Padrón, 1994, entre otros).

De ellos, coincidimos con Güell (2014) en que los grados de satisfacción alta se dan en dimensiones relacionadas con las condiciones de vida asociadas al empleo, la seguridad en el trabajo, la seguridad en el empleo, la libertad profesional y las vacaciones. Por el 
contrario, entre las dimensiones que producen una baja satisfacción entre los docentes con relación a la carrera realizada se encontrarían: el bajo prestigio profesional, la falta de recursos y formación, la baja retribución salarial y las posibilidades de ascenso.

Profundizando en la carrera del profesorado de Educación Física, nos interesa detenernos en estudios como el de Moreno, Sicilia y Águila (2000), que pretende determinar los motivos de la elección para la carrera del maestro de Educación Física, analizar las dificultades encontradas en la carrera y reflexionar sobre la importancia social del maestro; y el realizado años más tarde por Díaz y Barroso (2008), que trata de conocer la satisfacción del profesorado de Educación Física en Educación Secundaria desde cuestiones relacionadas con las condiciones de trabajo y la labor docente. Ambas investigaciones, contextualizadas en la comunidad autónoma de Andalucía (España), exponen la necesidad de ampliar los resultados ofrecidos realizando estudios a nivel nacional y, además, nos llevan a comprender que entre los aspectos más relevantes para conocer el grado de satisfacción del profesorado de Educación Física con la carrera realizada se encuentran: la formación inicial recibida (en la que se destacan los motivos por la elección de la carrera y la percepción de la formación inicial); las posibilidades de acceso a la función docente; y las vivencias en los centros.

\section{Objetivos}

De acuerdo con los últimos estudios y las limitaciones reseñadas en los mismos, nos planteamos como objetivo general de este trabajo conocer cuál es el grado de satisfacción del profesorado de Educación Física con la carrera realizada. El rasgo diferenciador del estudio que presentamos en relación a la bibliografía existente es que analiza la satisfacción de este grupo de profesores atendiendo a tres factores que se consideran clave para determinar esta variable: la satisfacción con la formación inicial recibida, el acceso a la labor profesional y la experiencia en el centro. Analizando estos tres factores nos marcamos como objetivos específicos: a) conocer las diferencias en la satisfacción profesional de los egresados en Educación Física atendiendo a la variable género; y b) indagar en las diferencias en relación a la satisfacción profesional de los profesionales de Magisterio de Educación Física y los de Ciencias de la Actividad Física y el Deporte.

\section{Metodología}

\subsection{Fases de la investigación}

El estudio realizado se asienta en una metodología de investigación mixta y ha sido elaborado en cinco fases. La investigación comenzó con la caracterización de los sujetos que participaron en la muestra y la elaboración de un cuestionario sobre diferentes aspectos relacionados con la carrera de Educación Física. Uno de ellos pretendía conocer el grado de satisfacción de los egresados con la carrera realizada. Posteriormente, se administraron los cuestionarios, por parte del equipo de investigación, en 14 Facultades de Educación españolas, durante el curso académico 2014/2015. En una fase posterior, y tras un primer análisis descriptivo de los datos, descubrimos que era necesaria la ampliación de la información recogida en relación a la pregunta de investigación realizada desde una mirada cualitativa. Para ello, se confeccionó un guion de entrevista estructurada y se recogieron datos de 20 participantes de la muestra, entre los meses de febrero y junio 
de 2018. Tras el análisis de los datos cualitativos, procedimos a realizar una discusión de la información recogida y a confrontarla con los datos de otras investigaciones. La última fase culminó con la presentación del informe de investigación que mostramos en las siguientes líneas.

\subsection{Participantes}

El estudio ha sido realizado con un total de 491 docentes de Educación Física (58,9\% hombres y 41,1\% mujeres), que finalizaron sus estudios entre los años 2000 y 2015. De ellos, 291 participantes son egresados de Facultades de Ciencias de la Actividad Física y el Deporte y 192 de Facultades de Educación. La mayoría de los encuestados tiene una edad inferior a 30 años (91,7\%) y fueron estudiantes de las localidades de Albacete, Barcelona, Córdoba, Huesca, La Laguna, León, Lleida, Madrid, Murcia, Segovia, Valencia, Valladolid, Vitoria y Zamora (Tabla 1).

Tabla 1. Distribución de la muestra por localidades

\begin{tabular}{lrrrr}
\hline & FreCUENCIA & PORCENTAJE & $\begin{array}{l}\text { PoRCENTAJE } \\
\text { VÁLIDO }\end{array}$ & $\begin{array}{c}\text { PoRCENTAJE } \\
\text { ACUMULADO }\end{array}$ \\
\hline Segovia & 21 & 4,3 & 4,4 & 4,4 \\
Albacete & 62 & 12,6 & 12,9 & 17,3 \\
Granada & 1 & 0,2 & 0,2 & 17,5 \\
Madrid & 6 & 1,2 & 1,3 & 18,8 \\
Valencia & 6 & 1,2 & 1,3 & 20,0 \\
Valladolid & 25 & 5,1 & 5,2 & 25,3 \\
Barcelona & 66 & 13,4 & 19,8 & 39,0 \\
Vitoria & 30 & 6,1 & 6,3 & 45,3 \\
Córdoba & 24 & 4,9 & 5,0 & 50,3 \\
León & 43 & 8,8 & 9,0 & 59,3 \\
Huesca & 59 & 12,0 & 12,3 & 71,6 \\
La Laguna & 8 & 1,6 & 1,7 & 73,3 \\
Lleida & 60 & 12,2 & 12,5 & 85,8 \\
Murcia- UCAM & 49 & 10,0 & 10,2 & 96,0 \\
Zamora & 15 & 3,1 & 3,1 & 99,2 \\
Zaragoza & 2 & 0,4 & 0,4 & 99,6 \\
Murcia - UMU & 2 & 0,4 & 0,4 & 100,0 \\
Total & 49 & 97,6 & 100,0 & \\
Perdidos sistema & 12 & 2,4 & & \\
Total & 491 & 100,0 & & \\
\hline Fuente: Elaboración propia. & & & & \\
\end{tabular}

\subsection{Instrumentos para la recogida de datos}

Como explicábamos anteriormente, la toma de datos se realizó mediante dos instrumentos: un cuestionario y una entrevista cerrada.

En relación al cuestionario, para recoger la valoración que los 491 docentes hicieron de la satisfacción con la carrera realizada se formuló la siguiente pregunta: ¿̨cuál es el grado de satisfacción con la carrera realizada? Para la respuesta a esta pregunta se utilizó una escala tipo Likert de diez puntos (0-10), donde "o" es "mínima satisfacción” y “10" es "máxima satisfacción”.

Este instrumento fue complementado con una entrevista semiestructurada que se realizó a 20 personas de la misma muestra del procedimiento cuantitativo (10 hombres y 10 mujeres), que tienen una edad media de 26 años y que son egresados, diez de ellos de Magisterio de Educación Física y otros diez de Ciencias de la Actividad Física y el 
Deporte. Para su selección, hemos tomado como referencia las diez localidades que tienen un mayor índice de participación en la muestra cuantitativa (Segovia, Albacete, Valladolid, Barcelona, Vitoria, Córdoba, León, Huesca, Lleida y Murcia) y hemos seleccionado al menos una persona de cada una de ellas, siendo más numerosas las entrevistas recogidas de la localidad de Segovia (5), Valladolid (4), León (2), Lleida (2) y Barcelona (2).

Para elaborar el guion de la entrevista se procedió a reformular la pregunta que guía la investigación tratando de conocer qué elementos subjetivos condicionaban la respuesta cuantitativa de los participantes y poder profundizar sobre los mismos (Anexo 1).

Durante todo el proceso de recogida de datos se ha velado por el anonimato de las personas que colaboran en el estudio y la confidencialidad de informaciones personales que pudieran perjudicarles, que han sido eliminadas del informe final.

\subsection{Análisis de los datos}

Los datos cuantitativos fueron analizados mediante el paquete estadístico SPSS 20.0 desde las variables: sexo y tipo de estudios. Puesto que el objetivo de la investigación era conocer el grado de satisfacción de los egresados de Educación Física con la carrera realizada y solo disponíamos de una pregunta en el cuestionario, decidimos realizar un análisis descriptivo de los datos, a nivel exploratorio, para conocer si existían diferencias iniciales.

Los datos cualitativos, recogidos en las entrevistas individuales, fueron transcritos y posteriormente analizados a través del programa ATLAS.ti. Para ello, primero realizamos una lectura de cada entrevista de forma individual y a continuación, buscamos conexiones entre las veinte entrevistas realizadas. Así, descubrimos tres núcleos de significado, que aparecían en todas ellas y daban sentido al análisis cualitativo: la satisfacción con la formación inicial recibida, el acceso a la labor profesional y la experiencia en el centro. Desde estos núcleos hicimos un análisis de las entrevistas atendiendo a las variables género y estudios, para poder establecer comparaciones y diferencias. Además, tuvimos en cuenta los diferentes temas que aparecían bajo los tres núcleos desde estas variables, un aspecto que nos ha permitido profundizar en cada uno de ellos.

Las citas literales recogidas de las entrevistas serán presentadas en el informe utilizando las variables: sexo, edad, estudios. Para el sexo utilizaremos las siglas $\mathrm{H}$ (hombres), $\mathrm{M}$ (mujeres). Para los estudios recurriremos a las siglas EMEF (Estudiantes de Magisterio de Educación Física) y ECAFID (Estudiantes de Ciencias de la Actividad Física y el Deporte). La edad se verá representada a través de su correspondiente dígito.

Los resultados serán presentados en dos apartados coincidentes con los objetivos del estudio: grado de satisfacción en función de la variable "sexo" y grado de satisfacción en función de la variable "estudios". Dentro de cada apartado abordaremos los tres ejes que hemos tenido en cuenta para el conocimiento de la satisfacción con la carrera realizada, centrados en la formación inicial recibida, el acceso al mundo laboral y la experiencia profesional. 


\section{Resultados}

\subsection{Grado de satisfacción en función de la variable "sexo"}

El análisis descriptivo de los datos nos permite comprobar como la media del grado de satisfacción de los hombres es de 6,02, mientras que la de las mujeres es de 6,30 (Tabla 2). Teniendo en cuenta que las desviaciones típicas de ambos grupos no presentan grandes diferencias, podemos concluir que no se muestran diferencias entre la satisfacción que manifiestan los hombres con la carrera realizada y la que presentan las mujeres, estando en ambos casos en un nivel medio.

Tabla 2. Estadísticos descriptivos del grado de satisfacción según el sexo

\begin{tabular}{lcc}
\hline & HOMBRES & MUJERES \\
\hline Media & 6,02 & 6,30 \\
Mediana & 7,00 & 7,00 \\
Desviación típica & 2,415 & 2,355 \\
Mínimo & 0 & 1 \\
Máximo & 10 & 10 \\
\hline
\end{tabular}

Fuente: Elaboración propia.

Cuando analizamos los datos a través del gráfico de puntuaciones, observamos que un amplio porcentaje de hombres sitúa sus respuestas por encima de los 7 puntos, pero otro grupo considerable lo hace por debajo del 5, incluso algunos puntúan su grado de satisfacción con la carrera realizada con o puntos (Figura 1). No ocurre lo mismo en el caso de las mujeres, que puntúan su satisfacción de 1 a 10 puntos, situándose un mayor número de respuestas entre los 7 y los 8 puntos.

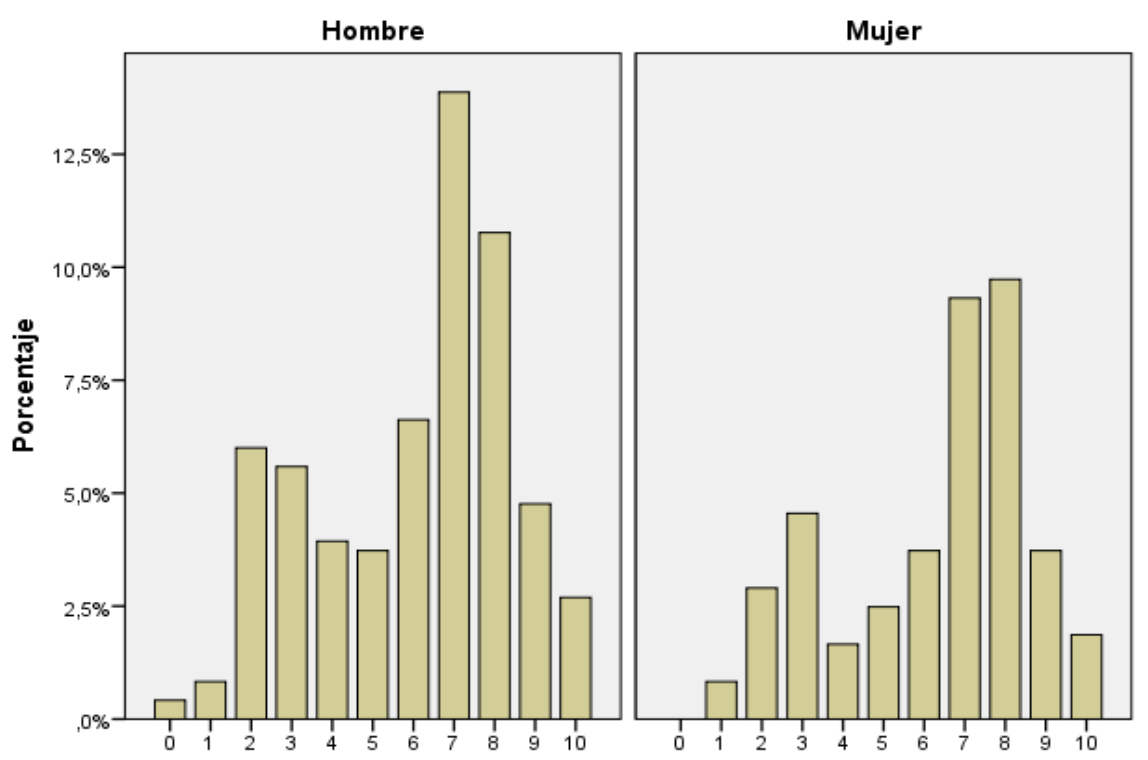

Figura 1. Puntuaciones sobre el nivel de satisfacción entre hombres y mujeres Fuente: Elaboración propia.

Sin embargo, las motivaciones que mueven la respuesta de cada uno de los participantes de acuerdo al sexo, contempla múltiples variables personales que deben ser analizadas. Em algunos casos, dichas variables están influídas por percepciones que parten de la educación recibida en espacios formales e informales. Así, si nos adentramos en los datos 
recogidos en las entrevistas, veremos cómo las motivaciones de hombres y mujeres por la elección de la carrera son completamente diferentes. Mientras el grupo masculino admite que eligió esta profesión porque le gustaba la actividad física y por las experiencias deportivas previas que había tenido en su trayectoria vital:

Elegí formarme en INEF porque desde siempre he tenido una vinculación especial con el deporte. Mi familia me lo inculcó desde niño y para mí la actividad física es un modo de vida. (H, 32 años, ECAFID)

Para la mayoría de las mujeres su elección viene determinada por la referencia de modelos docentes de Educación Física a lo largo de su trayectoria académica y por la vocación hacia el Magisterio. Sólo dos de las mujeres entrevistadas admite que eligió esta carrera por preferencias deportivas y esta decisión es la última de entre sus motivaciones.

Desde que comencé la universidad tenía especial interés en la especialidad de Educación Física, esta motivación venía precedida por varios maestros que tuve en Educación Primaria y Secundaria de esta asignatura además de mi vinculación con el deporte. (M, 25 años, EMEF)

En ambos casos, valoran la formación recibida de forma muy positiva, cumpliendo con sus expectativas antes de comenzar la carrera. Los factores que condicionan esta respuesta son la calidad de la formación inicial, la acertada adecuación de los contenidos y la buena relación con el profesorado universitario.

Como aspectos positivos del grado destaco: la gran profesionalidad de muchos de los profesores que tuve, así como su manera cercana de enseñarnos y querer transmitirnos sus conocimientos, con el fin de que les aprendiéramos, les pusiéramos en práctica y a su vez creáramos nuestra propia manera de enseñar. (H, 28 años, EMEF)

A pesar de ello, cinco hombres y seis mujeres de entre los entrevistados expresan que hubiera sido necesario aumentar el número de prácticas a lo largo de la carrera y haber adquirido una mayor formación en lengua extranjera, uno de los aspectos que consideran fundamentales para el acceso a la función docente.

De la formación recibida echo en falta más asignaturas que se dediquen exclusivamente a la Educación Física de modo práctico (ya que la E.F. —especialmente en Primaria- se centra en el juego y en lo práctico más que en la teoría) y menos que se dediquen exclusivamente a la teoría deportiva. Además, se necesita mayor formación en inglés. Después de terminar la carrera de Educación Física tuve que estudiar el C1, que es lo que me ha permitido trabajar a día de hoy como professor. (H, 25 años, EMEF)

En todos los casos, el sistema de oposición al cuerpo docente resulta ser una barrera importante para tener una satisfacción plena con la carrera elegida.

Antes de estudiar para ser maestro sabemos que tenemos que opositar, pero no siempre se tiene claro lo difícil que es encontrar trabajo. Las oposiciones son muy duras, da la sensación en ocasiones de pérdida de tiempo, somos demasiados para pocas plazas. Muchas veces sientes impotencia, dan ganas de tirar la toalla. (M, 28 años, EMEF)

Una vez superado el acceso a la función docente, hombres y mujeres admiten estar satisfechos con su elección. Entre los aspectos más destacados en esta satisfacción se encuentran, para las mujeres, el trato con los niños. Ocho de las entrevistadas convienen que el Magisterio es una profesión vocacional.

Uno de los aspectos positivos de esta profesión es la gratificación que recibes cada vez que un niño ha aprendido algo gracias a ti, el cómo evolucionan día a día y sin olvidar que todos los días aprendes algo nuevo de ellos, un aspecto que nos hace crecer como personas y como maestros. Es una profesión vocacional. (M, 25 años, EMEF) 
Además, las entrevistadas manifiestan que recurren con frecuencia a la pasión que sienten por la infancia y la educación, ante las dificultades que viven en el día a día, provocadas principalmente por el continuo cambio de destino.

El Magisterio es una carrera de fondo, sin duda. Yo creo que la gente que seguimos con ello es porque tenemos verdadera pasión por la educación y por los niños. Desde luego, todo aquel que cree que los maestros viven bien porque tienen un buen sueldo y muchas vacaciones no saben nada de la carrera docente. El desgaste que genera encontrar una plaza y vivir a merced del destino que te dan en septiembre no es nada fácil, pero cuando llegas al aula y estás con los alumnos, el sufrimiento merece la pena. (M, 28 años, EMEF)

En el caso de los hombres, la satisfacción — cuando ya han accedido al mundo laboralviene condicionada por: las posibilidades de formación posteriores, el buen trato con los compañeros y los recursos económicos.

Desde que estoy trabajando en un centro, mi satisfacción profesional viene condicionada por el destino, las relaciones con los compañeros del centro y el tiempo libre del que dispongo para seguir formándome. Nuestra profesión es una carrera de fondo y en ocasiones no está suficientemente pagada. (H, 31 años, ECAFID)

Hombres y mujeres, además, echan en falta una mayor consideración social de su profesión, sobre todo por parte de las familias.

Un aspecto negativo de esta carrera es que la sociedad no sabe valorar en muchos casos nuestra profesión, pues ven al maestro de Educación Física como el menos importante, pues estamos todo el día en chándal y somos el que juega con sus hijos. Las familias no ven más allá del estereotipo y realmente no saben todo lo que aprenden y desarrollan con nosotros. (H, 27 años, EMEF)

Además, los hombres, suelen manifestar su disconformidad con el estatus marginal de la Educación Física, la falta de recursos en los centros, la ausencia de posibilidades para la formación continua dentro del área y el limitado número de horas en el que enseñan la matéria. Este último aspecto, les convierte durante la mayor parte de la jornada en maestros generalistas, algo para lo que dicen no estar suficientemente formados y que les lleva a tener insatisfacción con la experiencia laboral.

\subsection{Grado de satisfacción en función de la variable "estudios"}

Desde la variable "estudios", observamos en el análisis descriptivo como la media de las respuestas de los egresados de Magisterio es de 6,04, mientras la de los egresados de Ciencias de la Actividad Física y el Deporte es de 6,20 (Tabla 4). A nivel puramente descriptivo y a la espera de realizar análisis inferenciales, teniendo en cuenta que las desviaciones típicas son parecidas, podemos concluir que no existen diferencias entre la percepción de ambos grupos con la carrera realizada.

Tabla 4. Estadísticos descriptivos del grado de satisfacción según los estudios

\begin{tabular}{lcc}
\hline & EMEF & ECAFID \\
\hline Media & 6,04 & 6,20 \\
Mediana & 7,00 & 7,00 \\
Desviación típica & 2,591 & 2,255 \\
Mínimo & 1 & 0 \\
Máximo & 10 & 10 \\
\hline
\end{tabular}

Fuente: Elaboración propia.

Esa misma conclusión podemos extraerla a través del gráfico de puntuaciones de cada uno de los grupos (Gráfico 2), a pesar de que se observa cómo los egresados de Ciencias de la 
Actividad Física y el Deporte tienen un mayor grado de satisfacción con la carrera que los egresados de Magisterio de Educación Física, ya que un porcentaje amplio de participantes del primer grupo otorgan puntuaciones de 7 a 10 puntos.

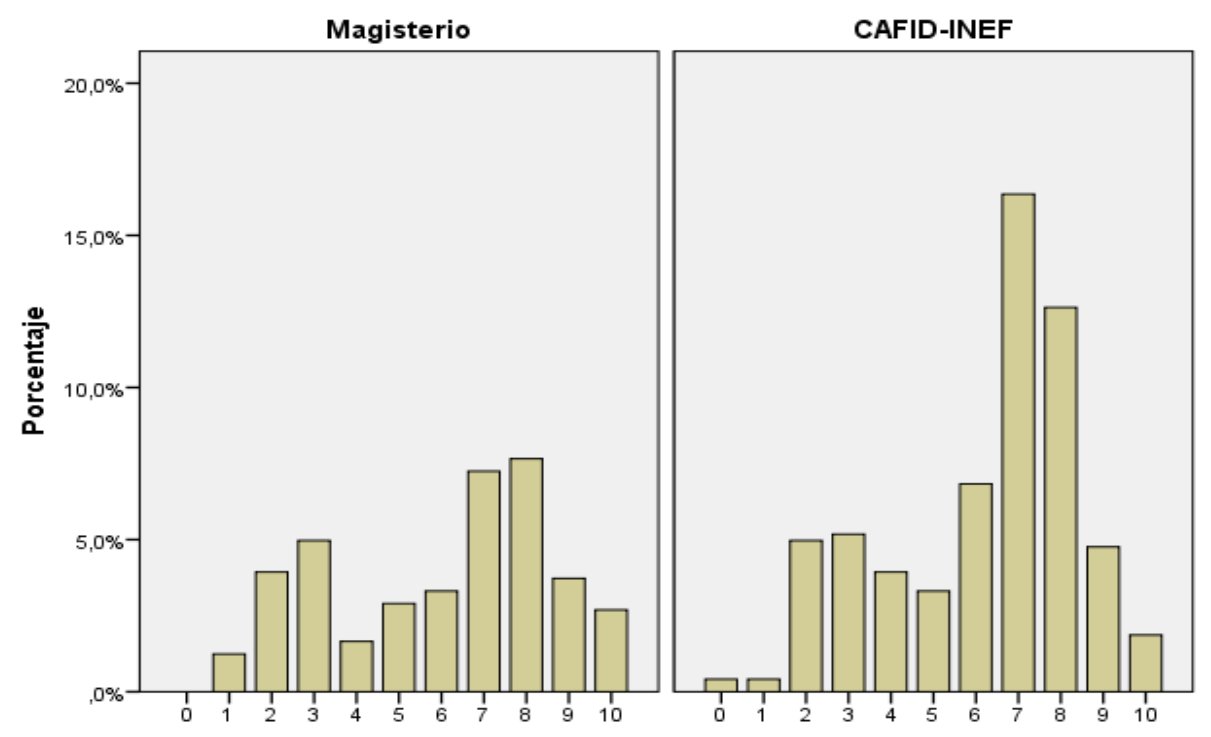

Gráfico 2. Puntuaciones EMEF y ECAFID del grado de satisfacción con la carrera Fuente: Elaboración propia.

Cuando contrastamos estos datos con las repuestas que los participantes ofrecen en las entrevistas, distinguimos que existe una correspondencia directa. Adentrándonos en sus narraciones podemos considerar que la elección de la carrera, en el caso de estudiantes de Magisterio de Educación Física, viene condicionada por un mayor número de factores externos, como el no haber podido acceder a otras titulaciones que exigían mayor nota de corte, la proximidad a la vivienda familiar o por ser el instrumento para acceder a otras titulaciones vinculadas, como el Grado en Ciencias de la Actividad Física y el Deporte. Además, los maestros de Educación Física expresan otras motivaciones internas como, por ejemplo, el gusto por la docencia y la influencia de modelos familiares y profesionales vinculados con la misma.

La elección de estudiar Magisterio de Educación Física vino motivada por varios factores: el primero fue que era la titulación que más me gustaba dentro de las ofrecidas por la Universidad de Valladolid; el segundo es que no me daba la nota de la selectividad para hacer INEF y esta carrera me ofrecía una formación parecida para trabajar en algo relacionado con los niños y el deporte; y la tercera razón es que yo siempre he querido ser maestro, así que tenía claro que mis estudios universitarios tenían que estar relacionados con la profesión docente. (H, 31 años, EMEF)

Las motivaciones en el caso de estudiantes de Ciencias de la Actividad Física y el Deporte tienen un carácter más interno y están relacionadas con el mundo del deporte. Los participantes expresan que el gusto por la actividad física y su relación con el deporte a lo largo de su trayectoria biográfica, son dos factores determinantes para la elección de la carrera.

Tenía muy claro que quería estudiar INEF y que me quería dedicar a la actividad física profesionalmente, porque desde siempre me ha gustado el deporte. Saqué buena nota en las pruebas de acceso y mis padres querían que hubiera hecho otra carrera. Mi padre es médico y 
siempre quiso que hiciera Medicina, pero a mí me gustaba INEF y lo hice en Madrid. (M, 29 años, ECAFID)

Tanto los profesionales vinculados con el Magisterio de Educación Física como aquellos formados en Ciencias de la Actividad Física y el Deporte, admiten que la formación inicial recibida cumplió con sus expectativas, a pesar de que las respuestas ofrecidas por los participantes muestran una clara diferencia entre las exigencias de una titulación y la de otra. Mientras los egresados de Magisterio consideran que la carrera no les resultó de gran dificultad:

La titulación no me resultó difícil. Tiene mucha carga de horas de trabajo en casa, de hacer proyectos, recensiones, lecturas, preparar prácticas... pero creo que el aprobado no es difícil de obtener. Lo que hay que tener claro es que, después, en las oposiciones, ese aprobado significa no tener plaza, por eso hay que intentar ir a por nota (y eso exige constancia, trabajo y mayor esfuerzo, algo a lo que no estamos acostumbrados). (H, 27 años, EMEF)

Los egresados de Ciencias de la Actividad Física y el Deporte explican que desde la prueba de evaluación de las aptitudes físicas — obligatoria para el acceso a la titulación—, vivieron un proceso de formación exigente que continuó a lo largo de la carrera.

Fueron años duros... tienes que tener una buena aptitud física y eso hay que demostrarlo desde que entras en la carrera. Además, las materias están vinculadas no solo con el deporte, sino que también el plan de estudios tiene una buena carga teórica. (H, 29 años, ECAFID)

Este aspecto hace que, en algunos casos, los maestros sientan cierta inferioridad respecto a los ECAFID, considerando a estos como profesionales con mayores competencias en el ámbito de la Educación Física y una mayor cualificación para el desarrollo de actividades profesionales vinculadas con el ámbito deportivo y ajenas a la docencia.

Aunque yo sentía que en la carrera de Magisterio me habian preparado bien para trabajar
en el colegio, cuando hice las prácticas me di cuenta que me faltaban competencias. Además,
yo creo que con Magisterio las salidas profesionales se reducen solo a las oposiciones y esto es
un problema, porque somos muchos para pocas plazas. (M, 29 años EMEF)

Los dos grupos de personas entrevistadas admiten que el momento de mayor insatisfacción profesional lo vivieron al terminar la carrera e intentar buscar trabajo. Mientras los maestros de Educación Física recurrieron en su mayoría al sistema de oposiciones como única salida profesional, un aspecto que critican por la dificultad de acceso y la masificación de opositores:

En cuanto al proceso de oposición en el que sigo estando inmersa lo he vivido de distintas maneras pero ninguna buena; siempre dicen que es una montaña rusa y nada más lejos de la realidad. Es un proceso tedioso con un alto coste a nivel psicológico y social. En mi caso nunca he llevado bien este proceso, no he sabido encontrar un punto de equilíbrio. (M, 28 años, EMEF)

Los ECAFID -que tampoco se sienten plenamente satisfechos con el sistema de oposiciónno solo recurren a esta vía para acceder al mundo laboral, sino que exploran otras salidas como la gestión deportiva, la preparación física, los programas de actividad física y salud o la promoción de empresas de servicios deportivos.

Me presenté una vez a las oposiciones, pero había pocas plazas y solo entré en listas. Al año siguiente encontré trabajo como monitor deportivo en una empresa privada y llevo varios años desempeñando mi trabajo allí(...). Una de las cosas que me gustan de mi trabajo es ganarme la vida haciendo deporte y enseñando a otros a tener una buena competencia física. (H, 30 años, ECAFID) 
Estas salidas profesionales serán condicionantes para que ambos grupos expresen su satisfacción en el puesto de trabajo. Mientras, los ECAFID, parecen estar satisfechos con su trabajo, independientemente de la salida profesional elegida.

Cuando terminé la carrera estudié el Master de Formación del Profesorado en Educación Secundaria y me presenté a oposiciones. Aprobé con una nota baja, pero desde entonces estoy trabajando (...). No puedo decir nada negativo de esta profesión, me siento afortunada de poder trabajar en lo que me gusta, además, a la mayoría de los alumnos les gusta la Educación Física y todos los años he conectado muy bien con ellos. (M, 30 años, ECAFID)

Los maestros y maestras expresan que las dificultades que se presentan en los primeros destinos (que suelen venir propiciadas por el trabajo en centros con una gran diversidad de alumnado, escasos recursos y professorado), la inexperiencia para impartir materias no relacionadas con la Educación Física y las escasas relaciones con compañeros (provocadas porque cubren bajas de otros docentes y no están mucho tiempo en el centro), son algunos aspectos que les llevan a tener una percepción negativa de la práctica ordinaria del aula.

\begin{abstract}
Durante el primer curso escolar me planteé varias veces si yo valía para ser profesora e incluso me planteé dejarlo, porque no me veía capaz. Si bien es cierto que los centros que me encontré fueron de todo menos fácil, tanto por la situación del alumnado como por la de los compañeros; a lo que hay que añadir el que fueron sustituciones cortas (de un mes) y a mitad de curso. Circunstancias que te hacen sentir y actuar no como una profesora sino como la sustituta de otra profesora, porque te ves condicionada a seguir su línea para perjudicar lo menos posible a los alunos. (M, 28 años, EMEF)
\end{abstract}

Ambos grupos de profesionales admiten que el trabajo en los centros educativos de Primaria y Secundaria, exige también disponer de una serie de competencias para las que no se sienten suficientemente formados. La resolución de los problemas y conflictos que se presentan en el aula, la colaboración con compañeros de otras asignaturas y la colaboración con la administración y otras instituciones para ofrecer al alumnado otras experiencias físicas, tanto dentro como fuera del horario escolar, son algunas de ellas.

\title{
6. Discusión y conclusiones
}

El estudio realizado avanza en mostrar que la satisfacción que perciben los docentes acerca de la carrera varía a nivel personal y viene condicionada por una serie de factores como la sensibilidad individual, las características personales, la trayectoria biográfica y otra serie de aspectos que configuran formas distintas de percibir la realidad laboral.

A nivel general, podemos decir que el profesorado de Educación Física tiene una satisfacción general con la carrera realizada media- alta. Entre los aspectos que la mayoría de participantes destaca como dimensiones que les reportan una alta satisfacción con la carrera elegida se encuentran: cuestiones vinculadas con la formación inicial recibida, de la que destacan —igual que lo hacen estudios como los de Pascual (2007), Folgueiras, Luna y Puig (2013) y Zurita y otros (2017)—: la calidad académica del profesorado universitario, la disposición para atender consultas, el trato cercano con los estudiantes y la práctica docente. Por el contrario, entre los aspectos que les llevan a tener una baja satisfacción con la carrera, los egresados señalan: la falta de un mayor número de experiencias prácticas en la carrera, la dificultad de acceso a la función docente, la escasa importancia académica que tiene la Educación Física para las familias y el reconocimiento social de su labor, aspectos que también han sido señalados en investigaciones como la de Díaz y Barroso (2008). 
Profundizando en los resultados ofrecidos, hacemos constar que si bien la variable "sexo", no presenta diferentes en las respuestas ofrecidas por los participantes del estudio a nivel cuantitativo, sí se hacen notar a nivel cualitativo, un detalle que también se aprecia en investigaciones como la de Güell (2014). Este hecho nos hace apoyar la teoría de Delgado y Zurita (2013), que mencionan la importancia que tiene en las investigaciones sobre el profesorado de Educación Física, tomar el género como categoría de análisis y utilizar datos cualitativos, ya que estos permiten abrir nuevas líneas de debate y conocer la influencia que la educación tradicional ha tenido en hombres y mujeres.

Atendiendo a este influjo, hemos podido constatar cómo la vocación y la pasión por la infancia aparecen como manifestaciones que motivan al género femenino para realizar la carrera de Educación Física; siendo el gusto por la actividad física y el deporte las motivaciones que mueven a los hombres a estudiar esta titulación. Estas motivaciones son en las que se refugian ambos grupos de profesionales para superar las barreras de acceso al mundo laboral y las que después valoran en su trabajo ordinario en el aula.

Junto a ellas, también apreciamos cómo el rol tradicional de género suele aparecer al comentar sus impresiones en la experiencia en los centros. Mientras las docentes viven la lejanía a la vivienda familiar y el continuo cambio de destino como problemas para tener una satisfacción profesional plena; en el caso de los hombres es la posibilidad de obtener una mejor formación y mejorar sus recursos económicos los aspectos que desdibujan una satisfacción con la carrera completa.

Avanzando en la discusión de los datos, en relación a las diferencias presentadas por los egresados de Ciencias de la Actividad Física y el Deporte y de Magisterio de Educación Física, hemos comprobado que, a pesar de que en análisis descriptivo no son amplias, sí se aprecian algunas cuando profundizamos en las los datos de las entrevistas, aceptando las conclusiones de estudios como los de Herrada y Herrada (2012) y Díaz y Barroso (2008). Estudios como el de Camina y Salvador (2007), coinciden con el nuestro en que los estudiantes de Magisterio de Educación Física eligen la carrera por: el gusto por la docencia, los contenidos específicos de la especialidad y la vocación. Estos profesionales admiten que después de la formación inicial, optan prioritariamente por el sistema de oposiciones como medio para acceder al mundo laboral, una experiencia que les genera sentimientos de estrés, disminución de la motivación y bajo interés por la carrera (Moreno, Sicilia y Águila, 2000).

Trabajos como el de Iznaola y Gabriel (2008) confirman nuestros resultados y nos llevan a apreciar cómo en la experiencia profesional, los maestros de Educación Física valoran como aspectos reseñables de su satisfacción con la carrera realizada: la posibilidad que les ofrece de enseñar, de ver la evolución física y madurativa de sus alumnos y de observar el disfrute del alumnado con la asignatura.

En el caso de los titulados de Ciencias de la Actividad Física y el Deporte, los profesionales admiten haber elegido la carrera por su vinculación con el deporte y explican que las exigencias en la formación inicial, a pesar de haber sido severas, les sirven tras la conclusión de la misma para tener mayores competencias profesionales y un abanico mayor de posibilidades en el mundo laboral, a pesar de que la participación en oposiciones de Secundaria suele ser la salida profesional más elegida por los participantes. 
Concluimos esta discusión dando respuesta a los objetivos que nos proponíamos al comienzo del artículo. Desde el primer objetivo, constatamos como el profesorado de Educación Física tiene motivaciones diferentes hacia la carrera en función del género, un aspecto que hace que valoren su nivel de satisfacción profesional de forma diferente. Para las mujeres, la vocación que dicen tener por la enseñanza y por la infancia son dos aspectos en los que se apoyan para superar las barreras que presenta el acceso al mercado laboral y que les hacen sentirse plenamente satisfechas cuando logran tener una plaza y trabajar en un centro. Como afirma una de las mujeres entrevistadas "cuando llegas al aula y estás con los alumnos, el sufrimiento merece la pena”. En el caso de los hombres, el gusto por el deporte y la actividad física son aspectos que les mueven no solo al elegir esta profesión, sino también a valorar positivamente esta elección. Su satisfacción con el mundo laboral está influida por el contacto con la actividad deportiva y la docencia.

En relación al segundo objetivo, constatamos cómo los profesionales que estudiaron Ciencias de la Actividad Física y el Deporte tienen una mayor satisfacción con la carrera realizada que los egresados de Magisterio de Educación Física. La salida profesional más valorada entre los maestros de Educación Física es el acceso a la función docente en el sistema público, un aspecto que critican por la masificación de opositores, la subjetividad en los procesos de oposición y los problemas emocionales y psicológicos que les supone esta prueba. Además, algunos maestros y maestras de Educación Física parecen no tener suficientes competencias cuando llegan por primera vez al aula. Como explicaba una de las participantes "durante el primer curso escolar me planteé varias veces si yo valía para ser profesora e incluso me planteé dejarlo, porque no me veía capaz”. Estas dudas e inquietudes vienen motivadas por la situación de itinerancia que viven en los primeros destinos, que no les permite tener un contacto prolongado con otros docentes ni establecer relaciones duraderas con el alumnado y las familias; las altas ratios profesor-alumno y los problemas del aula, pues las plazas ofrecidas se corresponden con los centros más conflictivos; y el papel de "sustituto", que les impide llevar sus propias programaciones en el aula.

Como conclusión general del artículo, podemos decir que el profesorado de Educación Física tiene un grado aceptable de satisfacción con la carrera realizada, siendo la formación inicial y el trabajo en el aula con el alumnado los aspectos que destacan como más positivos de su trayectoria y constituyendo la inserción profesional una fuerte barrera para estar plenamente satisfechos con el oficio docente.

Entre las limitaciones que presenta el estudio realizado, podemos citar: la información parcial aportada, la restricción que presenta la pregunta cuantitativa y la ausencia de un mayor número de datos, que nos lleve a percibir qué aspectos subjetivos y materiales condicionan a los profesionales de Educación Física para tener un mayor o menor grado de satisfacción con la carrera realizada. Estas limitaciones ayudan a presentar futuras líneas de trabajo. Entre ellas destacamos la posibilidad de realizar un análisis inferencial para conocer si las diferencias entre los grupos analizados son significativas; profundizar en las diferencias entre hombres y mujeres egresados de la titulación de Educación Física en relación a las condiciones profesionales experimentadas tras varios años de carrera, así como las barreras sufridas por su condición sexual; y conocer si las diferencias detectadas en la satisfacción profesional entre profesionales del Magisterio de Educación Física y Ciencias de la Actividad Física y el Deporte, aumentan con los años de experiencia, dadas 
las numerosas alusiones que el artículo hace a las oposiciones y a los periodos iniciales en el ejercicio profesional.

\section{Agradecimientos}

Apoyo/ financiación del trabajo: este estudio se ha llevado a cabo dentro del proyecto de $\mathrm{I}+\mathrm{D}+\mathrm{i}$ : "Las competencias docentes en la formación inicial del profesorado de educación física". Convocatoria de noviembre de 2013 del Programa Estatal de Investigación, Desarrollo e Innovación Orientada a los Retos de la Sociedad, en el marco del Plan Estatal de Investigación Científica y Técnica y de Innovación 2013-2016. Referencia: EDU 201342024-R. Duración: 3 años (2014-2016).

\section{Referencias}

Bimbela, J. L., Comas, A., Duran, A., Franco, J. J., Gálvez, S., González, M. P., Nueno, A., Notó, C., Oliver, D., Romero, M., Torres, M. y Villuendas, X. (2010). El bienestar del docente. Vivir bien educando: estrategias para conseguir satisfacción profesional y personal. Barcelona: Graó.

Caballero Rodríguez, K. (2002). El concepto de "satisfacción en el trabajo”. Profesorado, Revista de Currículum y Formación del Profesorado, 6(1-2), 1-10.

Cabrera, Alberto F., Weerts, David J. y Zulick, J. (2004). Encuestas a egresados: Tres fundamentos conceptuales en el seguimiento de egresados universitarios. En J. Vidal (Coord.), Métodos de análisis de la inserción laboral de los universitarios (pp. 55-80). Madrid: Ministerio de Educación, Cultura y Deporte.

Camina, A. y Salvador, M.I. (2007). Condicionantes y características de los estudiantes que inician magisterio. Estudio descriptivo y comparativo entre especialidades. Tendencias Pedagógicas, 12, 245-262.

Carbonero, M.A., Crespo, M.T., Martín, L.J., Ortega, O. y Sánchez, I. (1996). Satisfacción personal y académica de futuros profesores. Revista Interuniversitaria de Formación del Profesorado, 26, 153-161.

Delgado, M. A. y Zurita, F. (2003). Estudio de las teorías implícitas de la educación física en la formación inicial de los maestros en las diferentes especialidades de la Facultad de Ciencias de la Educación de la Universidad de Granada. Diferencias en función del género. Retos, Nuevas Tendencias en Educación Física, Deporte y Recreación, 5, 27-38.

Díaz González, P. y Barroso, J. (2008). Satisfacción del profesorado de educación física en educación secundaria. Revista digital Buenos Aires, 122.

Edmonds, R. (1982). Programs of school improvement. An overview. Educational Leadership, 40 (3), 4-11.

Fernández Sánchez, M. (1993). Encuesta al profesorado de primaria y secundaria de la enseñanza pública: Opiniones y actitudes ante sus condiciones de trabajo. Madrid: Centro de Publicaciones del Ministerio de Educación y Ciencia.

Folgueiras, P., Luna, E. y Puig, G. (2013). Aprendizaje y servicio: estudio del grado de satisfacción de estudiantes universitarios. Revista de Educación, 362, 159-185.

https://doi.org/10-4438/1988-592X-RE-2011-362-157

Gambau, V. (2015). Las problemáticas actuales de la educación física y el deporte escolar en España. Revista Española de Educación Física y Deportes, 411, 53-69. 
Gil-Flores, J., Álvarez Rojo, V., García Jiménez, E. y Romero Rodríguez, S. (2009). Evaluación de la formación universitaria a partir de las opiniones de los titulados incorporados al mundo laboral. Bordón, 61, 73-91.

Gonzalez-Riano, X. y Armesto-Fernandez, X. (2012). Minority language teaching and teacher satisfaction: The case of Asturias. Cultura y Educación, 24(2), 219-241.

Griffin, D. K. (2010). A survey of Bahamian and Jamaican teachers' level of motivation and job satisfaction. Journal of Invitational Theory and Practice, 16, 57-77.

Güell Malet, L. (2014). Estudio de la satisfacción laboral de los maestros. Tesis doctoral inédita, Barceloma: Universitat Internacional de Catalunya.

Herrada, R. I. y Herrada, G. (2012). Fin de las diplomaturas de magisterio: motivaciones, dificultad y satisfacción con la formación recibida. Tendencias Pedagógicas, 19, 175-188.

Herranz, M. y López Pastor, V. M. (2017). Perspectiva del alumno sobre su participación en los procesos de evaluación formativa y compartida en Educación Física en Primaria: un estudio de caso. EmasF, 48, 27-48.

Hortigüela, D., Abella, V. y Pérez-Pueyo, A. (2015). Percepciones del alumnado sobre la evaluación formativa: contraste de grupos de inicio y final de carrera. Revista de Docencia Universitaria, 13(3), 13-32.

Hortigüela, D. y Pérez-Pueyo, A. (2016). Percepción del alumnado de las clases de Educación Física en relación con otras asignaturas. Apunts, 123, 44-52.

Iznaola Cuscó, M.B. y Gabriel Wells, J. (2008). La satisfacción del profesor de Educación Física. Revista Educación Física y Deporte, 27 (2), 27-35. https://doi.org/10.5672/apunts.2014-0983.es.(2016/1).123.05

Jiménez, A., Terríquez, B. y Robles, F. J. (2011). Evaluación de la satisfacción académica de los estudiantes de la Universidad Autónoma de Nayari. Revista Fuente, 6, 46-56.

López-Pastor, V. M. y Palacios, A. (2012). Percepción de los futuros docentes sobre los sistemas de evaluación de sus aprendizajes. Revista Teoría de la Educación: Educación y Cultura en la Sociedad de la Información, 13(3), 317-341.

Martínez, L. F., Santos, M. L. y Castejón, F. J. (2017). Percepciones de alumnado y profesorado en Educación Superior sobre la evaluación en formación inicial en educación física. Revista RETOS, 32, 76-81.

Miles, W. L. (2010). Correlational study of leadership style and teacher job satisfaction in two head start programs. Recuperado de http://search.proquest.com/docview/761367262

Moreno Murcia, J. A., Sicilia Camacho, A. y Águila Soto, C. (2000). Percepciones de los futuros maestros especialistas en Educación Física sobre su carrera y su futuro profesional. Habilidad Motriz, 16, 15-27.

Padrón, M. (1994). Satisfacción profesional del profesorado. Tesis doctoral inédita. La Laguna: Universidad de La Laguna.

Palomares Aguirre, M. C. (2000). Variables que condicionan la satisfacción de los profesores que trabajan en colegios rurales agrupados. Bordón, 52(2), 213-227.

Pascual, I. (2007). Análisis de la satisfacción del alumno con la docencia recibida: un estudio con modelos jerárquicos lineales. RELIEVE, 13(1), 1-14.

Robbins, S.P. (1987). Comportamiento organizacional. Ciudad de México: Prentice-Hall Hispanoamericana. 
Sander, P., Stevenson, K., King, M. y Coates, D. (2000). University Student Expectations of Teaching. Studies in Higher Education, 25(3), 309-323. https://doi.org/10.1080/03075070050193433

Sonlleva Velasco, M., Martínez Scott, S. y Monjas Aguado, R. (2018). Los procesos de evaluación y sus consecuencias. Análisis de la experiencia del profesorado de Educación Física. Estudios Pedagógicos, 44(2), 329-351.

Thompson, D. G. (2008). Teacher job satisfaction and retention in a suburban Georgia school district. Recuperado de http://search.proquest.com/docview/815418238

Weinert, B. (1987). Manual de Psicología de la Organización. Barcelona: Herder.

Zurita Ortega, F., Viciana Garofano, V., Padial Ruz, R. y Cepero González, M. (2017). Niveles de satisfacción hacia el grado de maestro en alumnos de último curso. Profesorado, Revista de Currículum y Formación del Profesorado, 21(1), 349-367.

\section{Anexo 1. Guión de preguntas utilizado en la entrevista}

1. ¿Por qué elegiste estudiar la carrera de Educación Física?

2. ¿Cuáles son los aspectos positivos que destacarías de la formación recibida?

3. ¿Qué carencias detectas en tu formación como docente de Educación Física?

4. ¿Has realizado otras formaciones complementarias? ¿Cuáles?

5. ¿Cómo has vivido el proceso de oposición al cuerpo de maestros?

6. ¿Cómo valoras tus primeras experiencias laborales en un centro?

7. ¿Qué dificultades has encontrado desde la práctica en el aula?

8. ¿Qué características debe tener para ti un buen docente de Educación Física?

9. ¿Cuáles son los aspectos más positivos que destacarías de la profesión que has elegido? ¿y los negativos?

\section{Breve CV de los autores}

\section{Miriam Sonlleva Velasco}

Doctora en Educación por la Universidad de Valladolid; Licenciada en Pedagogía y Maestra de Educación Infantil y Primaria. Su formación académica se completa con un Máster en Psicología Infantil y otro en Investigación en Ciencias Sociales. Actualmente es miembro de las Sociedades Españolas de Historia de La Educación (SEDHE) y Patrimonio Histórico-Educativo (SEPHE). Participa en los Grupos de Investigación Reconocidos "Claves Históricas y Comparadas de la Educación. Género e Identidades" (Universidad Complutense de Madrid) e "Investigación e Innovación en Educación y Docencia Universitaria” (Universidad de Valladolid). Desde el año 2018 es contratada posdoctoral del Departamento de Pedagogía de la Universidad de Valladolid. Líneas de investigación: Historia de la Educación, Género y Formación Universitaria. ORCID ID: o000-0001-6268-8670. Email: miriam.sonlleva@uva.es 


\section{Suyapa Martínez Scott}

Doctora en Educación por la Universidad de Valladolid y Profesora Ayudante Doctora de la Facultad de Educación de Segovia (UVa). Sus líneas de investigación se centran en la formación del profesorado, la Educación para el Desarrollo y la Ciudadanía Global y la educación inclusiva. Miembro de diferentes $\mathrm{I}+\mathrm{D}+\mathrm{i}$, Proyectos de Innovación Docente y Grupos de Investigación reconocidos relacionados con la docencia universitaria así como miembro de la Red de Evaluación Formativa y Compartida. ORCID ID: 0000-0002-53237277. Email:suyapa@pdg.uva.es

\section{Roberto Monjas Aguado}

El historial científico de Roberto Monjas se desarrolla en paralelo a su labor como docente en la Universidad de Valladolid. Las líneas de investigación han sido la evaluación formativa, enseñanza deportiva y deporte escolar, deporte y educación en valores y la educación para el desarrollo. Su historial investigador tiene como punto culminante la tesis doctoral, en 2008, que aúna la investigación sobre enseñanza deportiva, evaluación y docencia universitaria. Los trabajos de investigación en los que ha intervenido exponen dos claras tendencias en su trayectoria profesional: la colaboración en proyectos regionales y nacionales, y su participación en proyectos de I+d+i. ORCID ID: 0000-00033572-1249. Email: rmonjas@mpc.uva.es 